\title{
ESTUDO DE CASO DO DESDOBRAMENTO DE METAS E GESTÃO DE DESEMPENHO DE PROJETOS NA ENGENHARIA DA PETROBRAS
}

\section{DEPLOYMENT OF GOALS AND PROJECT PERFORMANCE MANAGEMENT CASE STUDY IN THE ENGINEERING DEPARTMENT OF PETROBRAS}

\section{Antonio Carlos de Lemos Oliveira}

Mestre em Administração pelo Instituto de Pós-Graduação e Pesquisa em Administração da Universidade Federal do Rio de Janeiro - COPPEAD/UFRJ

Professor do Departamento de Engenharia Industrial da Universidade Federal do Rio de Janeiro UFRJ

E-mail: prof.lemos@gmail.com (Brasil)

\section{Leonardo Rabello da Silva}

Mestre em Engenharia de Produção pela Pontifícia Universidade Católica do Rio de Janeiro PUC/RJ

Professor convidado nas Universidades Gama Filho e Católica de Petrópolis

E-mail: leonardo.rsilva@ petrobras.com.br (Brasil)

\section{Luciana Mourao Temporal}

MBA em Gerenciamento de Projetos e Implementação de Empreendimentos pela Fundação Instituto de Administração da Universidade de São Paulo - FIA/USP

E-mail: ltemporal@ petrobras.com.br (Brasil)

\section{Glaiton Serafim}

MBA em Gerenciamento de Projetos e Implementação de Empreendimentos pela Fundação Instituto de Administração da Universidade de São Paulo - FIA/USP

E-mail: glaiton2005@yahoo.com.br (Brasil)

\section{Oscar Pedro Amaral Martins}

MBA em Gerenciamento de Projetos e Implementação de Empreendimentos pela Fundação Instituto de Administração da Universidade de São Paulo - FIA/USP

E-mail: oscarmartins@petrobras.com.br (Brasil) 


\title{
ESTUDO DE CASO DO DESDOBRAMENTO DE METAS E GESTÃO DE DESEMPENHO DE PROJETOS NA ENGENHARIA DA PETROBRAS
}

\begin{abstract}
RESUMO
O dinamismo das inovações tecnológicas e as constantes mudanças nos cenários geopolíticos mundiais têm causado crescente turbulência na maior parte dos ambientes em que as organizações da indústria do petróleo estão inseridas. Assim, torna-se vital para seu crescimento e sobrevivência estabelecer um processo de planejamento e gestão capaz de oferecer respostas flexíveis às diversas exigências do negócio. Nesse contexto, o presente trabalho tem como objetivo investigar quais práticas podem ser observadas no desdobramento de metas e gerenciamento de desempenho dos empregados da ENGENHARIA da Petrobras e comparar o desempenho de quatro unidades organizacionais (Unidade de Implementação de Empreendimento - UIE) por meio de alguns de seus indicadores com o resultado da avaliação de desempenho do corpo gerencial de cada uma delas, analisando-os à luz da base conceitual pesquisada. Os principais instrumentos de pesquisa utilizados nesse estudo de caso foram a análise documental e a realização de entrevistas semiestruturadas com os principais atores envolvidos no processo de formulação e implementação dos processos de planejamento e gestão da empresa, além da observação direta desses processos. $\mathrm{O}$ trabalho forneceu subsídios para ações já implementadas e para apresentação de propostas para a melhoria do processo de Gestão de Desempenho na ENGENHARIA da Petrobras.
\end{abstract}

Palavras-chave: Petrobras; Implementação de Empreendimentos; Balanced Scorecard; Gestão de Desempenho.

\section{DEPLOYMENT OF GOALS AND PROJECT PERFORMANCE MANAGEMENT CASE STUDY IN THE ENGINEERING DEPARTMENT OF PETROBRAS}

\begin{abstract}
The dynamic technology innovations and the constant changes in the world geopolitical scenarios have been creating turbulences in the majority of the environments in which the petroleum industry organizations are situated. Therefore, it is vital for the development and survival of these organizations that they establish a management and planning process capable of offering flexible answers to all challenges that arise in this business. Inside this context, this paper intends to investigate the ongoing techniques used for the deployment of goals and performance management of the employees who work for the engineering department of Petrobras, comparing the behavior of four organization structures named UIE - Unidade de Implementação de Empreendimento, considering some of their scores and the results of the existing individual management body of each of these structures. The main research instruments used in this case study were the documental analyses and semi-structured interviews with the main authors of the formulation and implementation process for the planning and management of the company, beyond the direct observation of these process. The paper accomplishes its goals and brings results and proposals that perceive a continuous development of the Performance Management system in the engineering department of Petrobras.
\end{abstract}

Keywords: Petrobras; Enterprise Implementation; Balanced Scorecard; Performance Management.

Revista de Gestão e Projetos - GeP, São Paulo, v. 1, n. 2, p 26-48, jul./dez. 2010. 


\section{INTRODUÇÃo}

A Petrobras tem realizado investimentos significativos em projetos, tanto nacionais quanto internacionais, em cumprimento ao seu Planejamento Estratégico 2020, em ambientes, muitas vezes, de grande complexidade e turbulência. A escassez de mão de obra e de infraestrutura operacional existentes em muitas das regiões em que a Petrobras pretende implementar seus projetos, aliada aos diferentes fatores culturais, sociais e climáticos e à alta demanda do mercado mundial por recursos logísticos e operacionais exclusivos da indústria do petróleo, configuram-se em variáveis de difícil controle e gestão, similarmente ao que Trist (1976) contextualizou como "ambientes turbulentos".

Nesse cenário, faz-se mister inovar no gerenciamento de projetos de petróleo e gás, sobretudo considerando-se o momento de forte demanda não facilmente suprida tanto pela escassez de recursos técnicos e humanos, quanto pelos relevantes custos operacionais e de implementação de empreendimentos.

Esta inovação, mais do que buscar a eficiência operacional e a superação da concorrência e das restrições ambientais por meio de melhorias incrementais nos sistemas de gerenciamento de projetos, deve buscar o alinhamento e a concretização do Planejamento Estratégico da organização, para assegurar as expectativas dos stakeholders, garantindo-lhes a rentabilidade de seus negócios e um posicionamento destacado no mercado.

\section{PLANEJAMENTO X IMPLEMENTAÇÃO: A SUPERAÇÃO DE UM DESAFIO}

De acordo com Charan e Colvin (1999), entre 70 e 90\% das organizações mundiais falham ao implementar a estratégia, que apesar de muitas vezes bem executada, perde seu valor por não ser reproduzida nos processos e na cultura da organização.

Ciente dos desafios existentes durante a implementação da estratégia, a Petrobras tem adotado o BSC (Balanced ScoreCard) como metodologia para a definição, o desdobramento, a aplicação e o controle da implementação da estratégia. Desenvolvido em 1992, por Kaplan e Norton para aperfeiçoar o sistema de medição dos aspectos intangíveis das organizações, o BSC tornou-se uma poderosa ferramenta tanto para descrever, como para implementar a estratégia de uma organização (Kaplan e Norton, 2000). 


\subsection{O BSC E O FATOR HUMANO ORIENTAM O GERENCIAMENTO DE PROJETOS}

No passado, o alinhamento dos empregados com a estratégia organizacional ou com os objetivos de um projeto não era fator crítico. Há um século, no apogeu da revolução da administração científica, as empresas dividiam complexas atividades de fabricação em sequências de tarefas muito mais simples. Gerentes e engenheiros industriais determinavam métodos de trabalho eficientes para cada tarefa e estabeleciam padrões de desempenho. Assim, as empresas tinham condições de contratar empregados sem escolaridade ou sem qualificações e treiná-los de forma estreita e profunda para a execução de tarefas relativamente simples e limitadas. $\mathrm{O}$ empregado ao executá-las repetidamente, desenvolvia destreza e habilidade naquela função.

Segundo afirmou Frederick Taylor - líder do movimento da Administração Científica "trabalhos simples para pessoas simples" (Taylor, 1990). Nesse ambiente, os empregados não precisavam compreender ou implementar a estratégia. Apenas deviam executar bem aquele trabalho limitado que os engenheiros e a gerência lhes atribuíram e para o qual foram treinados.

Atualmente, tal situação é insustentável com os constantes desafios existentes no caminho das organizações. Porter (1995) argumenta que "as atividades são as unidades básicas da vantagem competitiva" e que "a essência da estratégia está nas atividades - a opção de executar as atividades de maneira diferente ou de desempenhar atividades inovadoras".

Dessa forma, é fundamental que o empregado esteja preparado para interagir com o sistema de gerenciamento de projetos ao promover inovações ou ações que assegurem a eficiência operacional e garantam a rentabilidade para a companhia e para criar valores que atendam às expectativas dos clientes.

\subsection{A VALORIZAÇÃO DO FATOR HUMANO NA IMPLEMENTAÇÃO DO PLANEJAMENTO ESTRATÉGICO}

A Figura 1 apresenta, resumidamente, como o Planejamento Estratégico da Petrobras é refletido, sucessivamente, até o nível operacional no qual são delineadas as ações de cada funcionário, o que lhe permite conhecer e compreender a estratégia da companhia. Ainda, ao participar do planejamento e da definição de seus objetivos pessoais sob a tutoria do superior direto, promove-se o comprometimento do funcionário com as metas corporativas e setoriais.

Revista de Gestão e Projetos - GeP, São Paulo, v. 1, n. 2, p 26-48, jul./dez. 2010. 
Figura 1 - O BSC como um ciclo contínuo na definição da estratégia e da cadeia de valores.

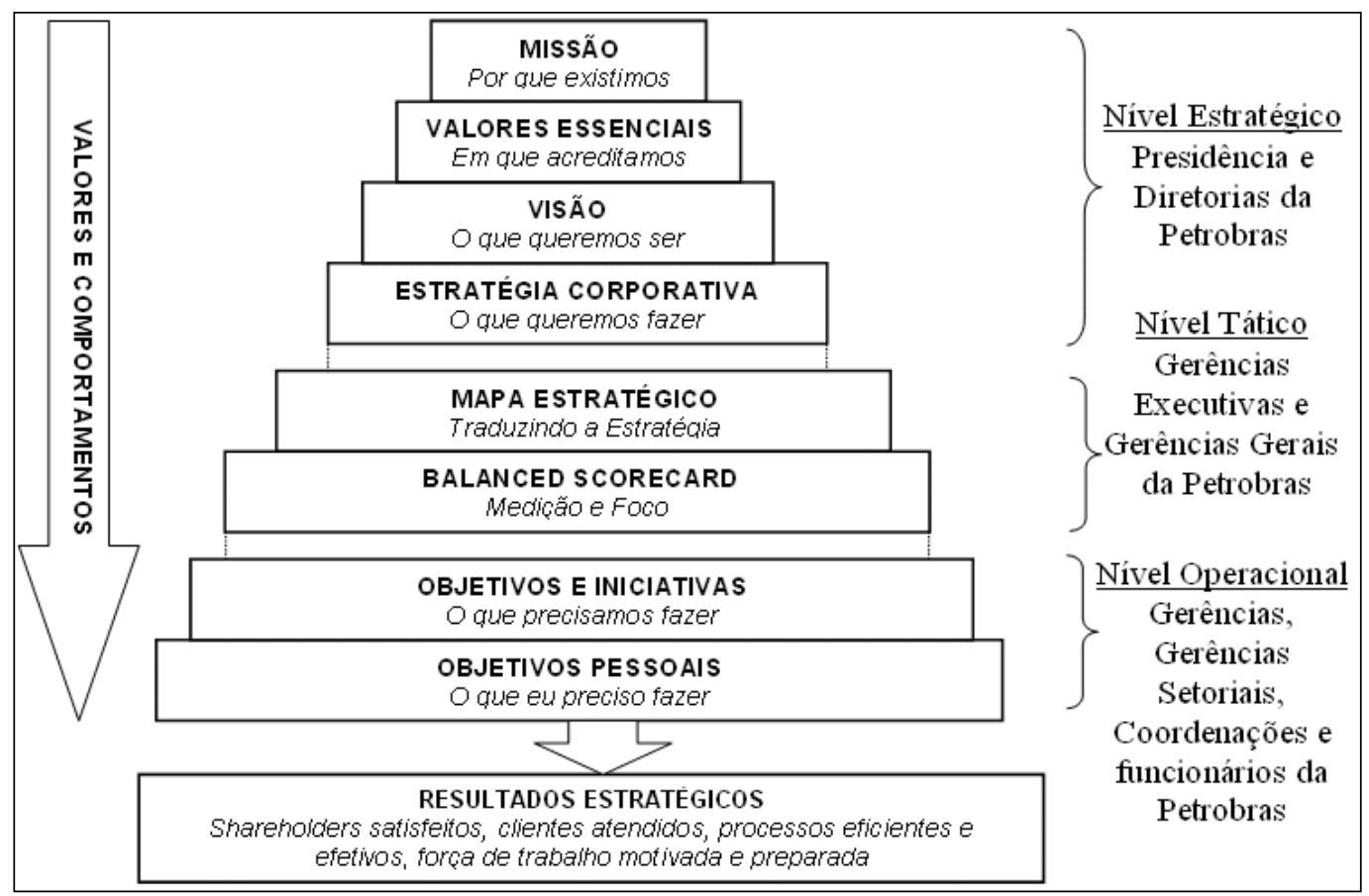

Fonte: Adaptado de Kaplan e Norton, 2000.

Os objetivos corporativos serão, então, segundo a metodologia do BSC, traduzidos em metas individuais que servirão de base para uma posterior análise da performance do funcionário e para a avaliação do seu comprometimento com o Planejamento Estratégico.

Mesmo pautada por um processo quantitativo atrelado às metas claras e aos resultados obtidos na execução de projetos ou processos, a avaliação de desempenho é fortemente influenciada pela subjetividade. 


\section{METODOLOGIA - O ESTUDO DE CASO}

O objetivo principal do presente estudo é o de comparar o desempenho da unidade organizacional (Unidade de Implementação de Empreendimento - UIE) por meio de alguns de seus indicadores com o resultado da avaliação de desempenho do corpo gerencial dessa unidade.

Nesse estudo de caso foram descritas e analisadas as práticas que vêm sendo utilizadas pelas diversas Gerências Gerais de Implementação de Empreendimentos (IE) da Unidade de Engenharia da Petrobras no sentido de refletir e implementar os objetivos estratégicos definidos corporativamente para suas Unidades de Implementação de Empreendimentos (UIE). Foi analisado o desdobramento de metas de quatro gerentes de UIE para os gerentes setoriais subordinados. Os autores buscaram evidências de alinhamento formal no Sistema de Gestão de Desempenho (GD), de metas do gerente da UIE para os gerentes setoriais imediatamente subordinados, a partir da seleção de seis indicadores de desempenho da UIE.

\subsection{PERGUNTAS A SEREM RESPONDIDAS}

As perguntas de pesquisa que orientaram o estudo de caso foram:

- Que práticas podem ser observadas no desdobramento de metas e gerenciamento de desempenho dos empregados da Unidade de Engenharia da Petrobras?

- Existem evidências de alinhamento formal no GD, de metas do gerente da UIE para os gerentes setoriais imediatamente subordinados?

- Existem diferenças entre o resultado global dessas UIE, medida pela verificação do desempenho consolidado no Painel de Desempenho da Unidade de Engenharia e o resultado da avaliação das metas do gerente da UIE e dos gerentes setoriais imediatamente subordinados no GD?

Revista de Gestão e Projetos - GeP, São Paulo, v. 1, n. 2, p 26-48, jul./dez. 2010. 


\subsection{TIPO DE PESQUISA}

Gil (1988, p. 147) afirma que "a parte mais complexa na redação de um projeto de pesquisa é constituída, geralmente, pela especificação da metodologia a ser adotada". Buscou-se fazer um estudo de caráter exploratório que, segundo Vergara (1997), é realizado em áreas nas quais há pouco conhecimento acumulado e sistematizado e, por sua natureza de sondagem, não comporta hipóteses que, todavia, poderão surgir durante ou ao final da pesquisa.

Schnelle (1967, p. 149) também afirma que, frequentemente, o estudo de caso é utilizado para investigar detalhes de um simples evento ou de um grupo de eventos correlatos com elevado grau de detalhes. Conforme Vergara (1997), o estudo do caso é circunscrito a uma ou poucas unidades, (...). Tem caráter de profundidade e detalhamento. Pode ou não ser realizado no campo.

\subsection{COLETA DOS DADOS}

Os procedimentos mais usados para a coleta de dados no estudo de caso são: a observação, a análise de documentos, a entrevista e a história de vida, sendo que, normalmente, utiliza-se mais de um procedimento. Os procedimentos utilizados nessa pesquisa para a coleta de dados foram: a análise de documentos e a entrevista. Para Yin (1989, p. 20), um ponto forte singular do estudo de caso é a possibilidade de lidar com uma extensa variedade de evidências, tais como documentos, artefatos, entrevistas e observações.

A coleta de dados sobre a empresa teve início com a pesquisa e a análise de documentos e de material produzido pela Unidade de Engenharia da Petrobras (relatórios, apresentações em PowerPoint, além de material abundante disponível na intranet e no site da empresa).

Foram entrevistados dois gerentes e um consultor envolvidos no processo de desdobramento de metas e gerenciamento de desempenho da Unidade de Engenharia da Petrobras.

A análise dos dados foi feita com dois objetivos básicos: responder às perguntas do estudo e traçar um paralelo, na medida do possível, entre o processo de desdobramento de metas e de gerenciamento de desempenho na Engenharia da Petrobras com a base conceitual apresentada na revisão de literatura.

Revista de Gestão e Projetos - GeP, São Paulo, v. 1, n. 2, p 26-48, jul./dez. 2010. 


\subsection{LIMITAÇÕES DO MÉTODO}

Segundo Vergara (1997, p. 59), “todo método tem possibilidades e limitações”. O presente estudo, por sua natureza exploratória e pelo método utilizado - o estudo de caso -, tem seus resultados restritos ao caso estudado, portanto, não permite generalizações.

\section{A ENGENHARIA}

A Engenharia é uma unidade organizacional da Diretoria de Serviços da Petróleo Brasileiro S.A. (Petrobras) e tem como finalidade implementar empreendimentos, prestar serviços de engenharia, em condições pactuadas com as áreas de negócios de Refino e Petroquímica, Exploração e Produção, Transporte Marítimo, Transporte Dutoviário, Gás e Energia e consolidar o conhecimento de gestão de empreendimentos acumulado na Companhia.

A estratégia da Engenharia, focada nos requisitos de seus clientes, é representada por objetivos estratégicos, refletidos em seu Mapa Estratégico, que possuem Indicadores de Desempenho e Iniciativas Estratégicas incorporados, os quais visam apoiar e monitorar seu alcance. O Mapa Estratégico e seus elementos são representados em uma ferramenta de apoio à gestão denominada Painel de Desempenho, utilizada para o acompanhamento contínuo dos resultados obtidos frente ao planejado.

A definição das metas tem por base os últimos resultados dos indicadores de cada unidade e o contexto atualizado, e é realizada por meio de sucessivas negociações entre os gerentes envolvidos até que os valores da integração de todas as metas das unidades e Gerências Gerais atendam às globais da Engenharia, conforme representado esquematicamente na Figura 2. As metas dos indicadores de desempenho são incluídas como resultados esperados, no Sistema de Gestão de Desempenho dos gerentes gerais (GG) e gerentes setoriais (GS). 
Figura 2 - Processo de desdobramento de metas e avaliação de desempenho da engenharia.

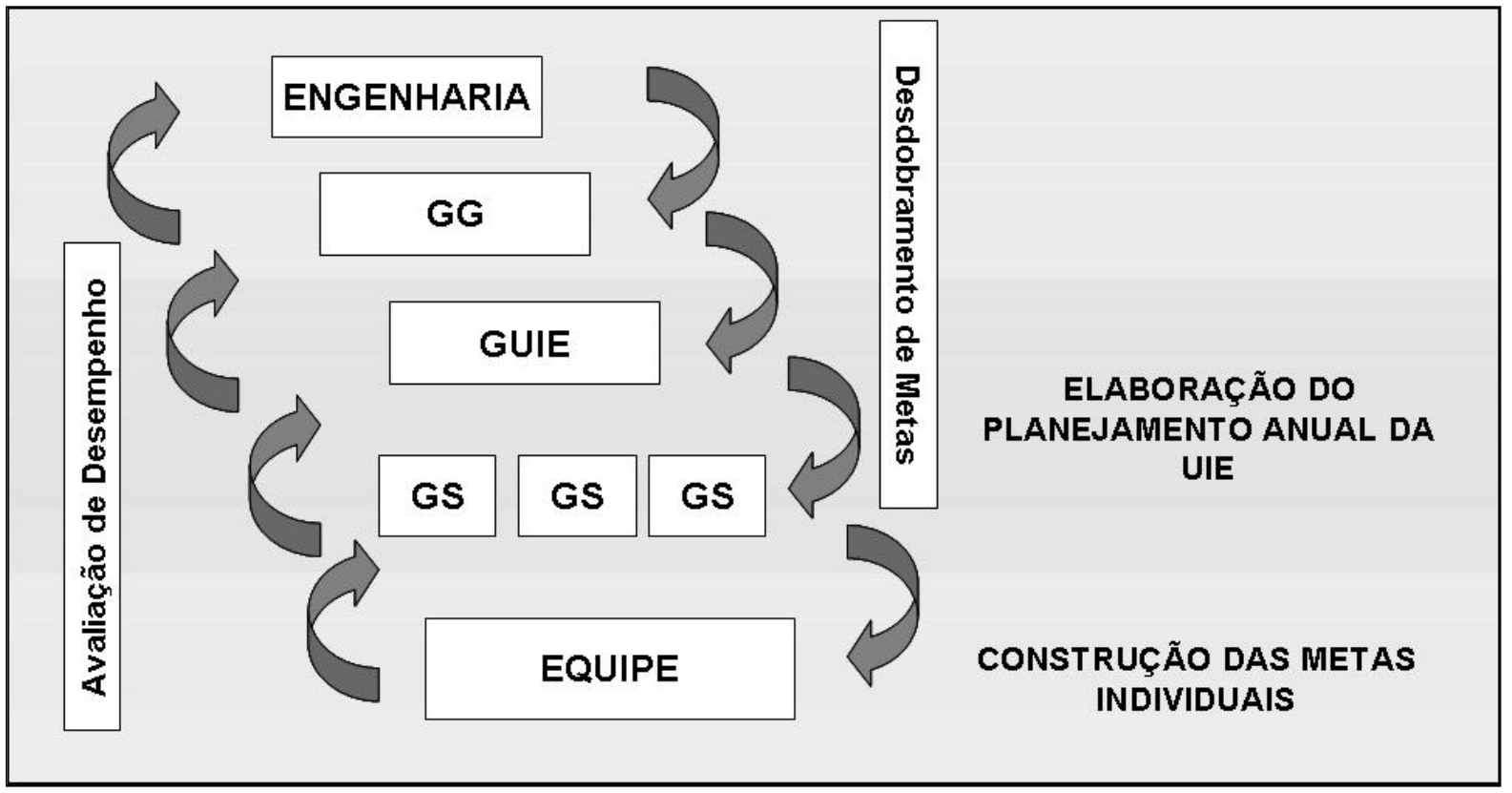

A partir desse nível de desdobramento, em cada gerência ocorre a elaboração de um plano de trabalho para o alcance das metas. As ações previstas são distribuídas na equipe de forma a identificar os responsáveis por sua execução. O conjunto de ações de cada integrante da equipe corresponde ao seu plano de trabalho com metas individuais.

\subsection{DESEMPENHO E DESDOBRAMENTO DE METAS NAS UNIDADES DE IMPLEMENTAÇÃO DE EMPREENDIMENTOS (UIE)}

A Engenharia segmentou o atendimento das Áreas de Negócio por meio da implantação de Gerências Gerais de Implementação de Empreendimentos (IE), compostas por diversas Unidades de Implementação de Empreendimentos (UIE), conforme representado esquematicamente na Figura 3. 
Figura 3 - Representação esquemática da Gerência Geral de IE, suas UIE com suas respectivas gerências setoriais.

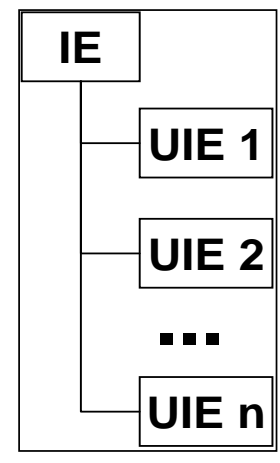

O estudo de caso se limitou a analisar duas UIE de duas Gerências Gerais de IE, uma com resultado global superior e outra com resultado global inferior. O resultado global foi medido por intermédio da verificação do desempenho consolidado dessas UIE em 2007 no Painel de Desempenho da Engenharia. Portanto, foram analisadas ao todo quatro UIE (por questões de sigilo, as Gerências Gerais de IE e as UIE não foram identificadas por suas denominações originais). As quatro UIE foram identificadas como: A1 e A2 que pertencem à mesma Gerência Geral de IE; e B1 e B2 que fazem parte de outra Gerência Geral de IE. O gerente da UIE foi denominado "Gerente" e a análise foi realizada a partir do desdobramento de metas do gerente da UIE para os gerentes setoriais subordinados. Esses gerentes setoriais foram nomeados "GS 1", "GS 2" e assim sucessivamente.

Como o estudo em questão relaciona-se com a gestão de projetos, decidiu-se selecionar e focar quatro indicadores do Painel de Desempenho da Engenharia que medem o desempenho em escopo, custo, prazo e qualidade, a chamada tríplice restrição (acrescida do quesito qualidade). Nas Tabelas 3, 8 e 13, a coluna "Geral" representa a média desses quatro indicadores. Foram acrescentados dois com o intuito de contextualizar e possivelmente trazer novos elementos que facilitem o entendimento do estudo de caso. Os dois indicadores são:

- Pessoas - que reflete o clima organizacional da UIE;

- Segurança - que reflete a taxa de frequência de acidentes com afastamento da UIE.

Revista de Gestão e Projetos - GeP, São Paulo, v. 1, n. 2, p 26-48, jul./dez. 2010. 
Os autores entendem que o sucesso no projeto com uma boa gestão de escopo, custo, prazo e qualidade não deve ser alcançado mediante o descaso ou pressão excessiva sobre pessoas ou sem a preocupação com as condições de segurança do trabalho na implementação dos empreendimentos.

Os autores buscaram evidências de alinhamento formal no Sistema de Gestão de Desempenho, de metas do gerente da UIE para os gerentes setoriais imediatamente subordinados, a partir da seleção de seis indicadores de desempenho da UIE.

\subsection{UNIDADES DE IMPLEMENTAÇÃO DE EMPREENDIMENTOS A1 E A2}

Na Tabela 1 são apresentados os resultados alcançados em 2007 pelas unidades de implementação de empreendimentos da Gerência Geral de IE " $A$ " com resultado global superior (denominado A1) e com resultado global inferior (denominado A2). A forma como os indicadores são calculados não são objeto desse estudo. Observa-se que a UIE A2 obteve um resultado inferior no indicador "Prazo" em comparação a UIE A1 e ambas tiveram um resultado semelhante em relação ao indicador "Custo".

Tabela 1 - Resultados das Unidades de Implementação de Empreendimentos A1 e A2 em 2007.

\begin{tabular}{|c|c|c|c|c|c|c|c|}
\hline & CUSTO & PRAZO & QUALIDADE & ESCOPO & PESSOAS & SEGURANÇA & GERAL \\
\hline A1 & $85 \%$ & $110 \%$ & $113 \%$ & $105 \%$ & $116 \%$ & $111 \%$ & $103 \%$ \\
\hline A2 & $87 \%$ & $52 \%$ & $101 \%$ & $110 \%$ & $101 \%$ & $101 \%$ & $87 \%$ \\
\hline
\end{tabular}

Com respeito às metas no Sistema GD para a UIE A1, é possível observar que em relação ao número de metas do Gerente (14), todos os nove Gerentes Setoriais obtiveram um número menor (média de 9), o que representa um número médio de metas de 35,7\%, menor que o do Gerente. Observa-se que o Gerente da UIE A1, quanto ao resultado esperado: superou em 3 das 14 metas a ele atribuídas, atingiu em 10 e atingiu parcialmente somente em uma. Isso se reflete em seu Resultado Consolidado de 101\%. Observa-se também que seu "Resultado Consolidado" foi menor que o de todos os Gerentes Setoriais diretamente subordinados a ele (Tabela 2).

Revista de Gestão e Projetos - GeP, São Paulo, v. 1, n. 2, p 26-48, jul./dez. 2010. 
Tabela 2 - Relatório de resultado de metas por empregado das UIE A1 e A2 em 2007.

\begin{tabular}{|c|c|c|c|c|c|c|c|c|c|c|c|c|c|c|}
\hline \multirow[b]{2}{*}{ UIE } & \multicolumn{2}{|c|}{$\begin{array}{c}\mathbf{N}^{\mathbf{o}} \\
\text { METAS }\end{array}$} & \multicolumn{2}{|c|}{$\begin{array}{c}\text { NÃO } \\
\text { ATINGIU } \\
(0 \%-69 \%)\end{array}$} & \multicolumn{2}{|c|}{$\begin{array}{c}\text { ATINGIU } \\
\text { PARCIALMENTE } \\
(70 \%-89 \%)\end{array}$} & \multicolumn{2}{|c|}{$\begin{array}{l}\text { ATINGIU } \\
(90 \%-109 \%)\end{array}$} & \multicolumn{2}{|c|}{$\begin{array}{l}\text { SUPEROU } \\
(110 \%-149 \%)\end{array}$} & \multicolumn{2}{|c|}{$\begin{array}{c}\text { SUPEROU } \\
(>150 \%)\end{array}$} & \multicolumn{2}{|c|}{$\begin{array}{c}\text { RESULTADO } \\
\text { CONSOLIDADO } \\
(\%)\end{array}$} \\
\hline & A1 & A 2 & A1 & A2 & A1 & A2 & A1 & A2 & A1 & A2 & A1 & A2 & A1 & A2 \\
\hline GERENTE & 14 & 16 & 0 & 2 & 1 & 0 & 10 & 13 & 3 & 1 & 0 & 0 & 101 & 96 \\
\hline GS1 & 8 & 9 & 0 & 1 & 0 & 1 & 2 & 6 & 6 & 1 & 0 & 0 & 108 & 91 \\
\hline GS2 & 8 & 11 & 0 & 1 & 0 & 3 & 2 & 5 & 6 & 2 & 0 & 0 & 108 & 90 \\
\hline GS3 & 8 & 7 & 0 & 1 & 0 & 2 & 1 & 4 & 7 & 0 & 0 & 0 & 109 & 83 \\
\hline GS4 & 8 & 2 & 1 & 0 & 0 & 0 & 1 & 1 & 6 & 1 & 0 & 0 & 104 & 103 \\
\hline GS5 & 8 & 6 & 0 & 1 & 0 & 1 & 2 & 4 & 6 & 0 & 0 & 0 & 108 & 90 \\
\hline GS6 & 12 & 4 & 0 & 0 & 0 & 0 & 6 & 4 & 6 & 0 & 0 & 0 & 105 & 98 \\
\hline GS7 & 8 & 1 & 0 & 0 & 0 & 0 & 1 & 1 & 7 & 0 & 0 & 0 & 109 & 90 \\
\hline GS8 & 13 & 3 & 0 & 0 & 0 & 1 & 6 & 1 & 7 & 1 & 0 & 0 & 105 & 92 \\
\hline GS9 & 8 & 7 & 0 & 0 & 0 & 1 & 2 & 5 & 6 & 1 & 0 & 0 & 108 & 102 \\
\hline
\end{tabular}

Já com respeito às metas no Sistema GD para a UIE A2, também é possível observar que em relação ao número de metas do Gerente (16), todos os nove Gerentes Setoriais obtiveram um número menor (média de 5,5), o que representa um número médio de metas 65,3\%, menor que o do Gerente. Observa-se que o gerente da UIE A2, quanto ao resultado esperado: superou em 1 das 16 metas a ele atribuídas, atingiu em 13 e não atingiu em 2. Isso se reflete em seu Resultado Consolidado de 96\%. Observa-se também que seu "Resultado Consolidado" foi menor que o de três Gerentes Setoriais diretamente subordinados a ele (Tabela 2).

Revista de Gestão e Projetos - GeP, São Paulo, v. 1, n. 2, p 26-48, jul./dez. 2010. 
A meta "Qualidade” não foi atribuída aos Gerentes das UIE A1 e A2 e, consequentemente, aos respectivos Gerentes Setoriais.

Em relação à UIE A1, também é um ponto de atenção o fato de as metas "Custo" e "Escopo" terem sido desdobradas para somente um dos nove Gerentes Setoriais. Ao analisar o conjunto selecionado de metas atribuídas por empregado, observou-se que, se o Gerente fosse avaliado somente pelos quatro indicadores-chave selecionados pelos autores desse estudo (custo, prazo, qualidade e escopo), ele teria obtido uma avaliação inferior ao registrado no Sistema GD. Ou seja, a quantidade de metas (14, conforme Tabela 2) serviu para melhorar o resultado consolidado na avaliação formal de desempenho no Sistema GD (Tabela 3).

Tabela 3 - Análise do conjunto selecionado de metas atribuídas por empregado em 2007 - UIE A1.

\begin{tabular}{|c|c|c|c|c|c|c|c|}
\hline & CUSTO & PRAZO & QUALIDADE & ESCOPO & PESSOAS & SEGURANÇA & GERAL \\
\hline GERENTE & $\begin{array}{c}\text { Atingiu } \\
\text { Parcialmente } \\
85 \%\end{array}$ & $\begin{array}{l}\text { Atingiu } \\
100 \%\end{array}$ & & $\begin{array}{c}\text { Atingiu } \\
100 \%\end{array}$ & $\begin{array}{c}\text { Superou } \\
110 \%\end{array}$ & $\begin{array}{c}\text { Atingiu } \\
100 \%\end{array}$ & $95 \%$ \\
\hline GS1 & & $\begin{array}{c}\text { Superou } \\
110 \%\end{array}$ & & & $\begin{array}{c}\text { Superou } \\
110 \%\end{array}$ & $\begin{array}{c}\text { Superou } \\
110 \%\end{array}$ & $110 \%$ \\
\hline GS2 & & $\begin{array}{c}\text { Superou } \\
110 \%\end{array}$ & & & $\begin{array}{c}\text { Superou } \\
110 \%\end{array}$ & $\begin{array}{l}\text { Supero } \\
110 \% \mathrm{u}\end{array}$ & $110 \%$ \\
\hline GS3 & & $\begin{array}{c}\text { Superou } \\
110 \%\end{array}$ & & & $\begin{array}{c}\text { Superou } \\
110 \%\end{array}$ & $\begin{array}{c}\text { Superou } \\
110 \%\end{array}$ & $110 \%$ \\
\hline GS4 & & $\begin{array}{c}\text { Superou } \\
110 \%\end{array}$ & & & $\begin{array}{c}\text { Superou } \\
110 \%\end{array}$ & $\begin{array}{c}\text { Superou } \\
110 \%\end{array}$ & $110 \%$ \\
\hline GS5 & & $\begin{array}{c}\text { Superou } \\
110 \%\end{array}$ & & & $\begin{array}{c}\text { Superou } \\
110 \%\end{array}$ & $\begin{array}{c}\text { Superou } \\
110 \%\end{array}$ & $110 \%$ \\
\hline GS6 & & $\begin{array}{c}\text { Superou } \\
110 \%\end{array}$ & & $\begin{array}{c}\text { Superou } \\
110 \%\end{array}$ & $\begin{array}{c}\text { Superou } \\
110 \%\end{array}$ & $\begin{array}{c}\text { Superou } \\
110 \%\end{array}$ & $110 \%$ \\
\hline GS7 & & $\begin{array}{c}\text { Superou } \\
110 \%\end{array}$ & & & $\begin{array}{c}\text { Superou } \\
110 \%\end{array}$ & $\begin{array}{c}\text { Superou } \\
110 \%\end{array}$ & $110 \%$ \\
\hline GS8 & $\begin{array}{c}\text { Atingiu } \\
90 \%\end{array}$ & $\begin{array}{c}\text { Superou } \\
110 \%\end{array}$ & & & $\begin{array}{c}\text { Superou } \\
110 \%\end{array}$ & $\begin{array}{c}\text { Superou } \\
110 \%\end{array}$ & $100 \%$ \\
\hline GS9 & & $\begin{array}{c}\text { Superou } \\
110 \%\end{array}$ & & & $\begin{array}{c}\text { Superou } \\
110 \%\end{array}$ & $\begin{array}{c}\text { Superou } \\
110 \%\end{array}$ & $110 \%$ \\
\hline
\end{tabular}

Revista de Gestão e Projetos - GeP, São Paulo, v. 1, n. 2, p 26-48, jul./dez. 2010. 
Em relação à UIE A2, também é um ponto de atenção o fato de que quatro Gerentes Setoriais não tenham nenhuma meta relacionada ao conjunto de seis selecionadas pelos autores. Ao analisar o conjunto selecionado de metas atribuídas por empregado, observou-se que, caso eles fossem avaliados somente pelos quatro indicadores-chave selecionados pelos autores desse estudo (custo, prazo, qualidade e escopo), todos teriam obtido uma avaliação inferior ao registrado no Sistema GD. Ou seja, as outras metas atribuídas serviram para melhorar o resultado consolidado de cada um dos envolvidos na avaliação formal de desempenho no Sistema GD. É possível observar também que para a meta referente ao "Prazo", o resultado da avaliação do Gerente foi pior que o de seus subordinados (Tabela 4).

Tabela 4 - Análise do conjunto selecionado de metas atribuídas por empregado em 2007 - UIE A2.

\begin{tabular}{|c|c|c|c|c|c|c|c|}
\hline & CUSTO & PRAZO & QUALIDADE & ESCOPO & PESSOAS & SEGURANÇA & GERAL \\
\hline GERENTE & $\begin{array}{c}\text { Atingiu } \\
\text { Parcialmente } \\
65 \% \\
\end{array}$ & $\begin{array}{c}\text { Não atingiu } \\
65 \% \\
\end{array}$ & & $\begin{array}{c}\text { Atingiu } \\
100 \%\end{array}$ & $\begin{array}{c}\text { Atingiu } \\
100 \%\end{array}$ & $\begin{array}{c}\text { Superou } \\
110 \%\end{array}$ & $76,67 \%$ \\
\hline GS1 & $\begin{array}{c}\text { Não atingiu } \\
50 \%\end{array}$ & $\begin{array}{c}\text { Atingiu } \\
\text { Parcialmente } \\
85 \%\end{array}$ & & $\begin{array}{l}\text { Atingiu } \\
100 \%\end{array}$ & $\begin{array}{c}\text { Superou } \\
110 \%\end{array}$ & $\begin{array}{c}\text { Atingiu } \\
110 \%\end{array}$ & $78,33 \%$ \\
\hline GS2 & $\begin{array}{l}\text { Não atingiu } \\
50 \%\end{array}$ & $\begin{array}{c}\text { Atingiu } \\
\text { Parcialmente } \\
80 \%\end{array}$ & & Atingiu & $\begin{array}{c}\text { Atingiu } \\
100 \%\end{array}$ & $\begin{array}{c}\text { Superou } \\
85 \%\end{array}$ & $76,67 \%$ \\
\hline GS3 & $\begin{array}{c}\text { Não atingiu } \\
50 \%\end{array}$ & $\begin{array}{c}\text { Atingiu } \\
\text { Parcialmente } \\
70 \%\end{array}$ & & & $\begin{array}{c}\text { Atingiu } \\
\text { Parcialmente } \\
70 \%\end{array}$ & $\begin{array}{l}\text { Atingiu } \\
100 \%\end{array}$ & $60,00 \%$ \\
\hline \multicolumn{8}{|l|}{ GS4 } \\
\hline GS5 & $\begin{array}{c}\text { Não atingiu } \\
50 \%\end{array}$ & $\begin{array}{c}\text { Atingiu } \\
\text { Parcialmente } \\
70 \%\end{array}$ & & & & & $60,00 \%$ \\
\hline \multicolumn{8}{|l|}{ GS6 } \\
\hline \multicolumn{8}{|l|}{ GS7 } \\
\hline \multicolumn{8}{|l|}{ GS8 } \\
\hline GS9 & & & & & & $\begin{array}{c}\text { Superou } \\
110 \%\end{array}$ & \\
\hline
\end{tabular}

Revista de Gestão e Projetos - GeP, São Paulo, v. 1, n. 2, p 26-48, jul./dez. 2010. 


\subsection{UNIDADES DE IMPLEMENTAÇÃO DE EMPREENDIMENTOS B1 E B2}

Na Tabela 5 são apresentados os resultados alcançados em 2007 pelas unidades de implementação de empreendimentos da Gerência Geral de IE "B" com resultado global superior (denominado B1) e com resultado global inferior (denominado B2).

Tabela 5 - Resultados das Unidades de Implementação de Empreendimentos B1 e B2 em 2007.

\begin{tabular}{|c|c|c|c|c|c|c|c|}
\hline & CUSTO & PRAZO & QUALIDADE & ESCOPO & PESSOAS & SEGURANÇA & GERAL \\
\hline B1 & $94 \%$ & $109 \%$ & $110 \%$ & $105 \%$ & $100 \%$ & $101 \%$ & $105 \%$ \\
\hline B2 & $76 \%$ & $99 \%$ & $94 \%$ & $93 \%$ & $91 \%$ & $71 \%$ & $90 \%$ \\
\hline
\end{tabular}

Observa-se na UIE B2 que, além de obter um resultado inferior em comparação a UIE B1, esta teve um resultado inferior nos indicadores "Pessoas" e "Segurança" (Tabela 5).

Com respeito às metas no Sistema GD para a UIE B1, é possível observar que os Gerentes Setoriais diretamente subordinados a ele obtiveram uma média de 8,7, o que representa um número médio de metas $45 \%$ maior que o do Gerente. Observa-se que o gerente da UIE B1 atingiu o resultado esperado em 5 das 6 metas a ele atribuídas e em uma, o atingiu parcialmente. Isso se reflete em seu Resultado Consolidado de 95\%. Observa-se também que seu "Resultado Consolidado" foi menor que o de todos os Gerentes Setoriais diretamente subordinados a ele (Tabela 6). 
Tabela 6 - Relatório de resultado de metas por empregado das UIE B1 e B2 em 2007.

\begin{tabular}{|c|c|c|c|c|c|c|c|c|c|c|c|c|c|c|}
\hline \multirow[b]{2}{*}{ UIE } & \multicolumn{2}{|c|}{$\begin{array}{c}\mathbf{N}^{\mathbf{o}} \\
\text { METAS }\end{array}$} & \multicolumn{2}{|c|}{$\begin{array}{c}\text { NÃO } \\
\text { ATINGIU } \\
(0 \%-69 \%) \\
\end{array}$} & \multicolumn{2}{|c|}{$\begin{array}{c}\text { ATINGIU } \\
\text { PARCIALMENTE } \\
(70 \%-89 \%) \\
\end{array}$} & \multicolumn{2}{|c|}{$\begin{array}{l}\text { ATINGIU } \\
(90 \%- \\
109 \%) \\
\end{array}$} & \multicolumn{2}{|c|}{\begin{tabular}{|c|} 
SUPEROU \\
$(110 \%-$ \\
$149 \%)$ \\
\end{tabular}} & \multicolumn{2}{|c|}{$\begin{array}{c}\text { SUPEROU } \\
(>150 \%)\end{array}$} & \multicolumn{2}{|c|}{$\begin{array}{c}\text { RESULTADO } \\
\text { CONSOLIDADO } \\
(\%) \\
\end{array}$} \\
\hline & B1 & B2 & B1 & B2 & B1 & B2 & B1 & B2 & B1 & B2 & B1 & B2 & B1 & B2 \\
\hline GERENTE & 6 & 6 & 0 & 0 & 1 & 0 & 5 & 0 & 0 & 0 & 0 & 0 & 95 & - \\
\hline GS1 & 3 & 28 & 0 & 0 & 0 & 0 & 3 & 28 & 0 & 0 & 0 & 0 & 103 & 100 \\
\hline GS2 & 10 & 16 & 0 & 0 & 0 & 3 & 8 & 7 & 2 & 6 & 0 & 0 & 102 & 99 \\
\hline GS3 & 10 & 16 & 0 & 0 & 0 & 3 & 9 & 7 & 1 & 6 & 0 & 0 & 101 & 99 \\
\hline GS4 & 8 & 13 & 0 & 0 & 0 & 3 & 7 & 4 & 1 & 6 & 0 & 0 & 101 & 98 \\
\hline GS5 & 11 & 13 & 0 & 0 & 0 & 3 & 9 & 7 & 2 & 3 & 0 & 0 & 101 & 96 \\
\hline GS6 & 8 & 13 & 0 & 0 & 0 & 3 & 6 & 7 & 2 & 3 & 0 & 0 & 101 & 96 \\
\hline GS7 & 8 & 13 & 0 & 0 & 0 & 3 & 6 & 5 & 2 & 5 & 0 & 0 & 102 & 99 \\
\hline GS8 & 8 & 8 & 0 & 0 & 0 & 0 & 7 & 4 & 1 & 4 & 0 & 0 & 102 & 104 \\
\hline GS9 & 11 & 16 & 0 & 0 & 0 & 3 & 10 & 7 & 1 & 6 & 0 & 0 & 101 & 99 \\
\hline GS10 & 10 & 10 & 0 & 0 & 0 & 2 & 9 & 5 & 1 & 3 & 0 & 0 & 102 & 98 \\
\hline
\end{tabular}

Já com relação às metas no Sistema GD para a UIE B2, é possível observar que os Gerentes Setoriais diretamente subordinados a ele tiveram uma média de 14,6, o que representa um número médio de metas $143 \%$ maior que o do Gerente. Observa-se que não são apresentados os resultados do Gerente da UIE B2. Ao investigar esse fato com o RH da Engenharia, verificou-se que o Gerente em questão foi transferido, no período em que as avaliações de desempenho deveriam ser realizadas. O Gerente não teve sua avaliação de desempenho realizada e a sua transferência fez com que o RH da Engenharia não tivesse mais acesso aos seus dados (Tabela 6).

Revista de Gestão e Projetos - GeP, São Paulo, v. 1, n. 2, p 26-48, jul./dez. 2010. 
Em relação à UIE $\mathrm{B} 1$, ao analisar o conjunto selecionado de metas atribuídas por empregado, observou-se que o Gerente não apresentava nenhuma meta desdobrada a partir dos indicadores-chave selecionados pelos autores desse estudo. Ao investigar esse fato com o RH da Engenharia, verificou-se que o Gerente atribuiu uma meta generalista que englobava todos os indicadores do Painel de Desempenho: "atingir as metas negociadas para os indicadores de desempenho". Isso justifica o fato de o Gerente ter 6 metas atribuídas no Sistema GD, bem menos que dos outros gerentes da Gerência Geral de IE A, também estudada (Tabela 7).

Tabela 7 - Análise do conjunto selecionado de metas atribuídas por empregado em 2007 - UIE B1.

\begin{tabular}{|c|c|c|c|c|c|c|c|}
\hline & CUSTO & PRAZO & QUALIDADE & ESCOPO & PESSOAS & SEGURANÇA & GERAL \\
\hline GEREN' & & & & & & & \\
\hline GS1 & & & $\begin{array}{c}\text { Atingiu } \\
105 \%\end{array}$ & & & & $105,00 \%$ \\
\hline GS2 & $\begin{array}{c}\text { Atingiu } \\
98 \%\end{array}$ & $\begin{array}{c}\text { Atingiu } \\
100 \%\end{array}$ & $\begin{array}{c}\text { Atingiu } \\
105 \%\end{array}$ & $\begin{array}{c}\text { Atingiu } \\
105 \%\end{array}$ & $\begin{array}{c}\text { Atingiu } \\
98 \%\end{array}$ & $\begin{array}{c}\text { Atingiu } \\
100 \%\end{array}$ & $102,00 \%$ \\
\hline GS3 & $\begin{array}{c}\text { Atingiu } \\
95 \%\end{array}$ & $\begin{array}{c}\text { Atingiu } \\
100 \%\end{array}$ & $\begin{array}{c}\text { Atingiu } \\
105 \%\end{array}$ & $\begin{array}{c}\text { Atingiu } \\
100 \%\end{array}$ & $\begin{array}{c}\text { Atingiu } \\
98 \%\end{array}$ & $\begin{array}{c}\text { Atingiu } \\
100 \%\end{array}$ & $100,00 \%$ \\
\hline GS4 & $\begin{array}{c}\text { Atingiu } \\
95 \%\end{array}$ & $\begin{array}{c}\text { Atingiu } \\
100 \%\end{array}$ & $\begin{array}{c}\text { Atingiu } \\
105 \%\end{array}$ & & $\begin{array}{c}\text { Atingiu } \\
98 \%\end{array}$ & & $100,00 \%$ \\
\hline GS5 & $\begin{array}{c}\text { Atingiu } \\
90 \%\end{array}$ & $\begin{array}{c}\text { Atingiu } \\
100 \%\end{array}$ & $\begin{array}{c}\text { Atingiu } \\
105 \%\end{array}$ & $\begin{array}{c}\text { Atingiu } \\
102 \%\end{array}$ & $\begin{array}{c}\text { Atingiu } \\
98 \%\end{array}$ & $\begin{array}{c}\text { Atingiu } \\
100 \%\end{array}$ & $99,25 \%$ \\
\hline GS6 & $\begin{array}{c}\text { Atingiu } \\
90 \%\end{array}$ & $\begin{array}{c}\text { Atingiu } \\
100 \%\end{array}$ & $\begin{array}{c}\text { Atingiu } \\
105 \%\end{array}$ & & $\begin{array}{c}\text { Atingiu } \\
98 \%\end{array}$ & & $98,33 \%$ \\
\hline GS7 & $\begin{array}{c}\text { Atingiu } \\
95 \%\end{array}$ & $\begin{array}{c}\text { Atingiu } \\
100 \%\end{array}$ & $\begin{array}{c}\text { Atingiu } \\
105 \%\end{array}$ & & $\begin{array}{c}\text { Atingiu } \\
98 \%\end{array}$ & & $100,00 \%$ \\
\hline GS8 & $\begin{array}{c}\text { Atingiu } \\
95 \%\end{array}$ & $\begin{array}{c}\text { Atingiu } \\
105 \%\end{array}$ & $\begin{array}{c}\text { Atingiu } \\
105 \%\end{array}$ & $\begin{array}{c}\text { Atingiu } \\
100 \%\end{array}$ & $\begin{array}{c}\text { Atingiu } \\
98 \%\end{array}$ & & $101,25 \%$ \\
\hline GS9 & $\begin{array}{c}\text { Atingiu } \\
95 \%\end{array}$ & $\begin{array}{c}\text { Atingiu } \\
100 \%\end{array}$ & $\begin{array}{c}\text { Atingiu } \\
105 \%\end{array}$ & $\begin{array}{c}\text { Atingiu } \\
105 \%\end{array}$ & $\begin{array}{c}\text { Atingiu } \\
98 \%\end{array}$ & $\begin{array}{c}\text { Atingiu } \\
100 \%\end{array}$ & $101,25 \%$ \\
\hline GS10 & $\begin{array}{c}\text { Atingiu } \\
98 \%\end{array}$ & $\begin{array}{c}\text { Atingiu } \\
100 \%\end{array}$ & $\begin{array}{c}\text { Atingiu } \\
105 \%\end{array}$ & $\begin{array}{c}\text { Atingiu } \\
105 \%\end{array}$ & $\begin{array}{c}\text { Atingiu } \\
98 \%\end{array}$ & $\begin{array}{c}\text { Atingiu } \\
100 \%\end{array}$ & $102,00 \%$ \\
\hline
\end{tabular}

Revista de Gestão e Projetos - GeP, São Paulo, v. 1, n. 2, p 26-48, jul./dez. 2010. 
Já em relação à UIE B2, ao analisar o conjunto selecionado de metas atribuídas por empregado, observou-se que caso eles fossem avaliados somente pelos quatro indicadores-chave selecionados pelos autores desse estudo (custo, prazo, qualidade e escopo), todos teriam obtido uma avaliação inferior ao registrado no sistema GD. Ou seja, o grande número de metas da UIE B2 serviu para melhorar o resultado consolidado de cada um dos envolvidos na avaliação formal de desempenho no Sistema GD (Tabela 8). É relevante o fato de que dois Gerentes Setoriais não tenham nenhuma meta relacionada ao conjunto de seis metas selecionadas pelos autores, assim como o fato de a meta "Prazo" não ter sido desdobrada para os Gerentes Setoriais e o empreendimento ter obtido um bom desempenho (99\%) conforme Tabela 5.

Tabela 8 - Análise do conjunto selecionado de metas atribuídas por empregado em 2007 - UIE B2.

\begin{tabular}{|c|c|c|c|c|c|c|c|}
\hline & CUSTO & PRAZO & QUALIDADE & ESCOPO & PESSOAS & SEGURANÇA & GERAL \\
\hline \multicolumn{8}{|c|}{ GERENTE } \\
\hline \multicolumn{8}{|l|}{ GS1 } \\
\hline GS2 & $\begin{array}{c}\text { Atingiu } \\
\text { Parcialmente } \\
76 \%\end{array}$ & & $\begin{array}{c}\text { Atingiu } \\
94 \%\end{array}$ & $\begin{array}{c}\text { Atingiu } \\
93 \%\end{array}$ & $\begin{array}{c}\text { Atingiu } \\
\text { Parcialmente } \\
85 \%\end{array}$ & $\begin{array}{c}\text { Atingiu } \\
\text { Parcialmente } \\
71 \%\end{array}$ & $87,67 \%$ \\
\hline GS3 & $\begin{array}{c}\text { Atingiu } \\
\text { Parcialmente } \\
76 \%\end{array}$ & & $\begin{array}{c}\text { Atingiu } \\
94 \%\end{array}$ & $\begin{array}{c}\text { Atingiu } \\
93 \%\end{array}$ & $\begin{array}{c}\text { Atingiu } \\
\text { Parcialmente } \\
85 \%\end{array}$ & $\begin{array}{c}\text { Atingiu } \\
\text { Parcialmente } \\
71 \%\end{array}$ & $87,67 \%$ \\
\hline GS4 & $\begin{array}{c}\text { Atingiu } \\
\text { Parcialmente } \\
76 \%\end{array}$ & & $\begin{array}{c}\text { Atingiu } \\
94 \%\end{array}$ & & $\begin{array}{c}\text { Atingiu } \\
\text { Parcialmente } \\
85 \%\end{array}$ & $\begin{array}{c}\text { Atingiu } \\
\text { Parcialmente } \\
71 \%\end{array}$ & $85,00 \%$ \\
\hline GS5 & $\begin{array}{c}\text { Atingiu } \\
\text { Parcialmente } \\
76 \%\end{array}$ & & $\begin{array}{c}\text { Atingiu } \\
94 \%\end{array}$ & $\begin{array}{c}\text { Atingiu } \\
93 \%\end{array}$ & $\begin{array}{c}\text { Atingiu } \\
\text { Parcialmente } \\
85 \%\end{array}$ & $\begin{array}{c}\text { Atingiu } \\
\text { Parcialmente } \\
71 \%\end{array}$ & $87,67 \%$ \\
\hline GS6 & $\begin{array}{c}\text { Atingiu } \\
\text { Parcialmente } \\
76 \% \\
\end{array}$ & & $\begin{array}{c}\text { Atingiu } \\
94 \% \\
\end{array}$ & $\begin{array}{c}\text { Atingiu } \\
93 \% \\
\end{array}$ & $\begin{array}{c}\text { Atingiu } \\
\text { Parcialmente } \\
85 \% \\
\end{array}$ & $\begin{array}{c}\text { Atingiu } \\
\text { Parcialmente } \\
71 \% \\
\end{array}$ & $87,67 \%$ \\
\hline \multicolumn{8}{|l|}{ GS7 } \\
\hline GS8 & & & $\begin{array}{c}\text { Atingiu } \\
94 \%\end{array}$ & & & & $94,00 \%$ \\
\hline GS9 & $\begin{array}{c}\text { Atingiu } \\
\text { Parcialmente } \\
76 \%\end{array}$ & & $\begin{array}{c}\text { Atingiu } \\
94 \%\end{array}$ & $\begin{array}{c}\text { Atingiu } \\
93 \%\end{array}$ & $\begin{array}{c}\text { Atingiu } \\
\text { Parcialmente } \\
85 \%\end{array}$ & $\begin{array}{c}\text { Atingiu } \\
\text { Parcialmente } \\
71 \%\end{array}$ & $87,67 \%$ \\
\hline GS10 & & & $\begin{array}{c}\text { Atingiu } \\
94 \%\end{array}$ & $\begin{array}{c}\text { Atingiu } \\
93 \%\end{array}$ & $\begin{array}{c}\text { Atingiu } \\
\text { Parcialmente } \\
85 \%\end{array}$ & $\begin{array}{c}\text { Atingiu } \\
\text { Parcialmente } \\
71 \%\end{array}$ & $93,50 \%$ \\
\hline
\end{tabular}

Revista de Gestão e Projetos - GeP, São Paulo, v. 1, n. 2, p 26-48, jul./dez. 2010. 


\section{ANÁl ISE DO CASO E CONCLUSÕES}

A cada dia, gestores percebem que os ativos mais importantes de suas organizações são as pessoas. Observa-se uma verdadeira revolução nas áreas relacionadas à gestão de recursos humanos. No entanto, algumas questões ficam no ar. Como as organizações devem proceder para gerenciar de forma estratégica seus colaboradores? Antes disso, o que realmente é esperado desses colaboradores e como pode ser criado um ambiente propício para que esses objetivos sejam atendidos?

Para responder às perguntas propostas no estudo de caso, por intermédio das entrevistas buscou-se descrever as práticas relacionadas ao desdobramento de metas e gerenciamento de desempenho dos empregados da Engenharia da Petrobras. Quanto às perguntas relacionadas à evidência de desdobramento e os resultados obtidos, os autores analisaram duas UIE de duas Gerências Gerais de IE, uma UIE com resultado global superior e outra com resultado global inferior. O resultado global foi medido com a verificação do desempenho consolidado dessas UIE em 2007 no Painel de Desempenho da Engenharia.

Em referência à quantidade de metas desdobradas, no caso dos empreendimentos ligados a Gerência Geral de IE “A”, observou-se um melhor resultado na UIE A1 que desdobrou um número maior de metas que na UIE A2 porém isso não foi confirmado para a Gerência Geral de IE "B" onde um melhor resultado foi observado na UIE que, comparativamente, desdobrou um menor número de metas. É razoável supor que não é a quantidade de metas desdobradas que garante o melhor resultado. Caberia aqui uma investigação mais profunda com o objetivo de apurar se existe alguma correlação de desdobramento de metas e de resultados obtidos para cada uma das Gerências Gerais de IE e se haveria alguma justificativa para diferenças entre as mesmas já que todas estão ligadas à mesma Unidade Organizacional (Engenharia).

Em relação ao conjunto de metas atribuídas ao empregado (vide Tabela 9), observou-se que, nas UIE A1 e B1, caso eles fossem avaliados somente pelos quatro indicadores-chave selecionados pelos autores desse estudo (custo, prazo, qualidade e escopo), todos teriam obtido uma avaliação semelhante ao registrado no Sistema GD. No caso das UIE A2 e B2 a avaliação cairia de "Atingiu (90\%-109\%)" para “Atingiu parcialmente (70\%-89\%)".

Revista de Gestão e Projetos - GeP, São Paulo, v. 1, n. 2, p 26-48, jul./dez. 2010. 
Tabela 9 - Resumo do desdobramento de metas.

\begin{tabular}{|c|c|c|c|c|c|}
\hline UIE & $\begin{array}{c}\text { Média de } \\
\text { metas } \\
\text { desdobradas } \\
\text { no GD (todas } \\
\text { as metas) } \\
\\
\text { a } \\
\end{array}$ & $\begin{array}{c}\text { Média do } \\
\text { Resultado } \\
\text { Consolidado do } \\
\text { Gerente e Gerentes } \\
\text { Setoriais no GD } \\
\text { (todas as metas) } \\
\text { a } \\
\end{array}$ & $\begin{array}{c}\text { Média de metas } \\
\text { selecionadas } \\
\text { desdobradas no } \\
\text { GD (escopo, } \\
\text { custo, prazo e } \\
\text { qualidade) } \\
\text { b } \\
\end{array}$ & $\begin{array}{l}\text { Média do Resultado Consolidado } \\
\text { do Gerente e Gerentes Setoriais } \\
\text { para as metas selecionadas } \\
\text { desdobradas no GD (escopo, } \\
\text { custo, prazo e qualidade) } \\
\text { b }\end{array}$ & Diferença \\
\hline A1 & 9,50 & $106,50 \%$ & 1,40 & $107,50 \%$ & $1,00 \%$ \\
\hline A2 & 6,60 & $93,50 \%$ & 1,30 & $70,33 \%$ & $-23,17 \%$ \\
\hline B1 & 8,45 & $101,00 \%$ & 3,09 & $100,91 \%$ & $-0,09 \%$ \\
\hline B2 & 13,82 & $98,80 \%$ & 1,54 & $88,85 \%$ & $-9,95 \%$ \\
\hline
\end{tabular}

Fonte: a - Tabelas 2 e 6; b - Tabelas 3, 4, 7 e 8 .

Nas UIE A1 e A2 houve um menor número de desdobramento de metas com resultados bastante diferenciados. No caso das UIE B1 e B2, a UIE que teve resultado global superior (B1 vide Tabela 5) foi a que desdobrou mais metas selecionadas (escopo, custo, prazo e qualidade). Na UIE B2, observou-se que o melhor resultado obtido (prazo - vide Tabela 5) foi o único que não foi desdobrado (vide Tabela 8). Em função da diversidade de resultados encontrados, é razoável supor que o simples desdobramento das metas não seja suficiente para garantir o resultado esperado. A qualidade do desdobramento é muito importante.

Para as UIE A1 (103,0\% x 107,5\%) e B1 (105,0\% x 100,9\%) que tiveram um resultado global superior, não há diferença significativa do resultado obtido pela UIE e pelos empregados no Sistema de Gestão de Desempenho (Tabela 10). 
Tabela 10 - Resultado da UIE x desdobramento de metas.

\begin{tabular}{|c|c|c|c|c|}
\hline UIE & $\begin{array}{c}\text { Resultado médio } \\
\text { de metas } \\
\text { selecionadas da } \\
\text { UIE (painel de } \\
\text { desempenho) }\end{array}$ & $\begin{array}{c}\text { Média do Resultado Consolidado } \\
\text { do Gerente e Gerentes Setoriais } \\
\text { para as metas selecionadas } \\
\text { desdobradas no GD } \\
\text { (escopo, custo, prazo e } \\
\text { qualidade) }\end{array}$ & $\begin{array}{c}\text { Média do } \\
\text { Resultado } \\
\text { Consolidado do } \\
\text { Gerente e Gerentes } \\
\text { Setoriais no GD } \\
\text { (todas as metas) }\end{array}$ & $\begin{array}{c}\text { Há diferença significativa } \\
\text { entre as metas da UIE } \\
\text { selecionadas no Painel de } \\
\text { Desempenho e as metas do } \\
\text { empregado selecionadas } \\
\text { no Sistema GD? }\end{array}$ \\
\hline A1 & $103,0 \%$ & $107,5 \%$ & $106,5 \%$ & $\begin{array}{c}\text { Sem diferença } \\
\text { significativa. }\end{array}$ \\
\hline A2 & $87,0 \%$ & $70,3 \%$ & $93,5 \%$ & Há diferença. \\
\hline B1 & $105,0 \%$ & $100,9 \%$ & $101,0 \%$ & Sem diferença \\
significativa.
\end{tabular}

Fonte: a - Tabelas 1 e 5; b - Tabelas 3, 4, 7 e 8; c - Tabelas 2 e 6.

No caso da UIE A2 (87,0\% x 70,3\%), que teve um resultado global inferior, houve diferença significativa do resultado obtido pela UIE e pelos empregados no Sistema de Gestão de Desempenho. Nesse caso, é razoável supor que, ou as metas da UIE foram mal desdobradas pelo Gerente para os Gerentes Setoriais, ou o Gerente foi mais rigoroso na avaliação dos Gerentes Setoriais. Mas isso não pode ser inferido somente com os dados disponíveis (Tabela 10).

Já para a UIE B2 $(90,0 \%$ x 88,8\%), que também teve um resultado global inferior, não houve diferença significativa do resultado obtido pela UIE e pelos empregados no Sistema de Gestão de Desempenho. Só há alguma diferença ao considerar todas as metas $(98,8 \%)$ dos empregados inseridas no Sistema de Gestão de Desempenho. O acréscimo de outras metas além das que os autores desse trabalho selecionaram favoreceu o resultado da avaliação de desempenho dos empregados ligados a essa UIE. É razoável supor que essas metas adicionais possivelmente mais fáceis de atingir, possam tirar o foco das metas críticas da UIE (escopo, custo, prazo e qualidade) (Tabela 10).

Revista de Gestão e Projetos - GeP, São Paulo, v. 1, n. 2, p 26-48, jul./dez. 2010. 
Em resumo, observou-se que nem sempre a avaliação dos empregados no Sistema GD reflete os resultados obtidos pela UIE. Os autores esperavam encontrar uma maior coerência entre o resultado obtido pela avaliação de desempenho das pessoas e a avaliação do resultado da UIE.

A partir da base conceitual disponível em Quinn et al. (2003), observou-se que a Engenharia pode obter uma série de benefícios significativos de crescimento e desenvolvimento por meio de um correto desdobramento de metas e consequente delegação de tarefas e responsabilidades para sua força de trabalho.

Também segundo a base conceitual disponível em Robbins (2002), a prática de feedback deve ser incentivada na Engenharia, principalmente para o corpo gerencial, pois isto as ajuda a perceber as discrepâncias entre o que tem sido feito e aquilo que precisa ser realizado para o alcance do objetivo.

É visível o esforço da Engenharia em reforçar a realização de fóruns, treinamentos de desdobramento de metas e desenvolvimento das competências individuais, principalmente para o público gerencial, o qual busca o fortalecimento da disseminação do processo de Gestão de Desempenho, com foco na sua relação entre o Balanced Scorecard (BSC) da Petrobras, o Plano Estratégico da Engenharia e as metas individuais de cada empregado.

Apesar disso, o processo de negociação entre gerentes e empregados parece ter áreas de oportunidade de melhoria, desde o alinhamento, acompanhamento e a avaliação das metas. Nesse estudo, os autores verificaram que muitas metas deixam de ser desdobradas para os gerentes, mas que curiosamente não deixaram de ser alcançadas no nível da UIE. Não foi objeto desse estudo verificar a qualidade da definição das metas mas foi recorrente a reclamação de que as metas desdobradas estão longe de ser específicas, mensuráveis, atribuíveis, realistas e com prazo definido.

\section{REFERÊNCIAS}

Charan, R. e Colvin, G. (1999). Why CEO's fail? Fortune Magazine, 139, 2.

Gil, A. C. (1988). Métodos e técnicas de pesquisa social. (2a. ed.) São Paulo: Atlas.

Kaplan, R. S. e Norton, D. P. (2000). Organização orientada para a estratégia. (4a. ed.). Rio de Janeiro: Campus.

Revista de Gestão e Projetos - GeP, São Paulo, v. 1, n. 2, p 26-48, jul./dez. 2010. 
Porter, M. E. (1995). Competitive advantage: creating and sustaining superior performance. New York: The Free Press.

Quinn, R. E.; Faerman, S. R.; Thompson, M. P. e McGrath, M. R. (2003). Competências gerenciais: princípios e aplicações. Rio de Janeiro: Elsevier.

Robbins, S. P. (2002). Comportamento organizacional. (9a. ed.), São Paulo: Prentice Hall.

Schnelle, K. E. (1967). Case analysis and business problem solving. New York: McGraw-Hill.

Taylor, F. W. (1990). Princípios da administração científica. (8a. ed.). São Paulo: Atlas.

Trist, E. L. (1976). Some concepts of planning. national seminar on long range planning. Perth, Australia: University of Western Australia.

Vergara, S. C. (1997). Projetos e relatórios de pesquisa em administração. São Paulo: Atlas.

Yin, R. K. (1989). Case study research: design and methods. (Vol. 5). Thousand Oaks, CA, USA: Sage Publications.

Data do recebimento do artigo: 13/07/2010

Data do aceite de publicação: 04/10/2010

Revista de Gestão e Projetos - GeP, São Paulo, v. 1, n. 2, p 26-48, jul./dez. 2010. 